\title{
Smoking habits and plasma lipid peroxide and vitamin E levels in never-treated first-episode
}

\section{patients with schizophrenia}

\author{
SCOTTISH SCHIZOPHRENIA RESEARCH GROUP
}

\author{
Background Lipid peroxidation may \\ be increased in schizophrenia, due to the \\ illness, lifestyle or medication.
}

\begin{abstract}
Aims To determine plasma lipid peroxide levels and serum vitamin $E$ and $A$ levels in first-episode never-treated people with schizophrenia and in controls.
\end{abstract}

\begin{abstract}
Method Thirty in-patients with a first episode of schizophrenia or

schizophreniform psychosis were recruited, as were controls matched for gender, age, smoking and dietary status. Blood samples were taken, smoking status was recorded and body mass index measured.
\end{abstract}

\begin{abstract}
Results There were no significant differences between patients and controls in plasma lipid peroxide levels. Seventythree per cent of the patients smoked. Patients who smoked had a higher mean lipid peroxide level than non-smokers. Seventy-seven per cent of patients and $70 \%$ of controls had a ratio of vitamin E to cholesterol of less than 5 . Body mass index was lower in patients than in controls.
\end{abstract}

\begin{abstract}
Conclusions As a result of the high prevalence of smoking this group shows increased lipid peroxidation. Low serum ratios of vitamin $E$ to cholesterol in both patients and controls suggest an unsatisfactory diet.
\end{abstract}

Declaration of interest The study was supported by an unrestricted grant from Eli Lilly.
It has long been suggested (Lohr, 1991) that lipid peroxidation may be increased in people with schizophrenia. Possibly they have increased oxidative stress or impaired antioxidant defence, or both (Mahadik \& Mukhergee, 1996). To date, all the studies except one have examined medicated patients. In these studies it was not clear whether the increased lipid peroxidation was associated with the illness itself or with antipsychotic drugs, which can be a source of free radicals (Chignell et al, 1985); but a study of first-episode never-medicated patients would eliminate the possibility of drugs as a source of free radicals. One such study (Mahadik et al, 1998) did find increased lipid peroxidation; however, the patients were not clearly matched with a comparison group. In the present study we measured plasma lipid peroxide levels and serum levels of the antioxidants, vitamins $\mathrm{E}$ and $\mathrm{A}$, in never-treated first-episode patients with schizophrenia and a wellmatched control group. We also recorded smoking habits and body mass index (BMI), a basic measure of nutritional status.

\section{METHOD}

\section{Subjects}

Patients were recruited from six hospitals in the West of Scotland. All fulfilled DSM-IV diagnostic criteria for either schizophrenia or schizophreniform psychosis (American Psychiatric Association, 1994). It was the first episode of illness for these patients, and the admission was their first to inpatient psychiatric care. None had ever received antipsychotic drugs. A control subject was recruited for each patient, matched for gender, age (within 5 years), smoking status (smoker/non-smoker) and dietary status (non-vegetarian/vegetarian).

The study was approved by the local research ethics committee for each hospital. A complete description of the study was given to each patient and control subject and written information was provided. Written informed consent was obtained.

\section{Assessment}

A blood sample was taken from patients and control subjects to measure plasma lipid peroxide and serum cholesterol, vitamin $\mathrm{E}$ and vitamin A. Blood samples were taken by syringe and dispensed into glass tubes containing tripotassium ethylene diamine tetraacetic acid (EDTA) solution (Becton Dickinson, Oxford). After centrifugation, carried out within $1 \mathrm{~h}$ of blood collection, $500 \mu \mathrm{l}$ aliquots of plasma were added to polypropylene screw-capped tubes containing $50 \mu$ ethanolic butylated hydroxytoluene (BHT) solution to give a final BHT concentration of $5 \mathrm{mmol} / \mathrm{l}$. These were mixed thoroughly and stored at $-20^{\circ} \mathrm{C}$ until analysis. The biochemist did not know whether blood samples were from patients or control subjects.

Plasma lipid peroxide was measured by the high-performance liquid chromatography (HPLC) method of Young \& Trimble (1991). Serum concentrations of vitamin E and vitamin A were measured by the method of Catignani \& Bieri (1983). Vitamin E status can be expressed either as the serum concentration or as the ratio of vitamin $\mathrm{E}$ to cholesterol in the serum. Vitamin $\mathrm{E}$ is transported within lipid particles in plasma. Differences in serum vitamin $\mathrm{E}$ concentration are influenced by variations in serum cholesterol levels, and therefore it is preferable to use this ratio rather than vitamin $\mathrm{E}$ concentration alone.

The patients' mental state was assessed using the Positive and Negative Syndrome Scale (PANSS) for schizophrenia (Kay et $a l, 1987)$ and the Clinical Global Impression (CGI) Scale (Guy, 1976). The assessments were carried out by psychiatrists trained in the use of the PANSS. The patients' height and weight were also measured.

\section{Statistical analysis}

Differences between patients and control subjects were assessed using McNemar's test for categorical variables and Student's paired $t$-test for continuous variables. Differences between unpaired groups were assessed using $\chi^{2}$ and unpaired $t$-tests. Correlation between variables was assessed using the Pearson product moment correlation coefficient. All $P$ values are two-tailed. 


\section{RESULTS}

\section{Social and demographic data}

Thirty patients were recruited between 1996 and 1998 (21 men, nine women). Twenty-one had schizophrenia and nine had schizophreniform psychosis. The men were younger (mean 28 years, s.d. 7 years) than the women (mean 33, s.d. 11) but this difference was not statistically significant. The mean age of the control subjects was 30 years (s.d. 7). Thirty-seven per cent of the patients lived on their own, $20 \%$ with parents, $17 \%$ with their spouse or partner and $26 \%$ had other living arrangements; jects were $30 \%, 13 \%, 27 \%$ and $30 \%$. Eighty per cent of patients and $52 \%$ of control subjects had never married (McNemar's test: $\chi^{2}=7.69$, d.f. $=1$, $P=0.006)$. Twenty-eight patients were non-vegetarian; two were vegetarian (i.e. did not eat meat).

\section{Blood test results}

Blood samples were taken from all patients and control subjects. Levels of plasma lipid peroxide, serum cholesterol, vitamin $\mathrm{E}$ and vitamin A are shown in Table 1. There were no significant differences between patients and control subjects in plasma lipid peroxide, serum vitamin $\mathrm{A}$ and the ratio of vitamin $\mathrm{E}$ to cholesterol, but serum vitamin $\mathrm{E}$ levels were lower in patients with schizophrenia. In $77 \%$ of patients and min $\mathrm{E}$ to cholesterol was less than 5, the minimum level said to be necessary to protect against heart disease (Hense et al, 1993).

The percentage of smokers in patients with schizophrenia (and therefore in control subjects also) was $73 \%$ (76\% male, $67 \%$ female). The mean number of the corresponding figures for control sub$70 \%$ of control subjects, the ratio of vita-

cigarettes smoked daily was 16 (s.d. 6); in control subjects it was 17 (s.d. 6).

Plasma lipid peroxide levels were higher in smokers than in non-smokers; this difference was statistically significant in patients with schizophrenia (mean $0.54 \mu \mathrm{mol} / \mathrm{l}$, s.d. 0.15 ) v. 0.40 (s.d. 0.16); difference in means 0.14 ; $95 \%$ CI $0.01-0.27$; unpaired $t$-test: $t=2.27$, d.f. $=28, P=0.03$ ) but not in control subjects $(0.56$ (s.d. 0.26) $v$. 0.47 (s.d. 0.12)).

\section{Body mass index}

Height and weight measurements were available for 24 pairs of patients and control subjects. There were no significant differences between patients and control subjects in mean height $(1.72 \mathrm{~m}$ (s.d. 0.08) v. 1.74 (s.d. 0.13)) but patients weighed less (mean $65.7 \mathrm{~kg}$ (s.d. 11.1) $v$. 75.0 (s.d. 149); mean of differences 8.46; $95 \%$ CI $0.91-16.00 ; t=2.32$, d.f. $=23$, $P=0.03$ ). The BMI (weight $(\mathrm{kg}) \div$ height $(\mathrm{m})^{2}$ ) was lower in patients (mean 22.2 (s.d. 3.6) v. 24.7 (s.d. 3.4); mean of differences 2.15; 95\% CI 0.06-4.25; paired $t$ test: $t=2.12$, d.f. $=23, P=0.04)$. Nineteen per cent of the patients were overweight (BMI 25-29.9) or obese (BMI $\geqslant 30$ ), compared with $44 \%$ of control subjects (not statistically significant).

\section{Mental state}

Mean (s.d.) scores of the patients on the positive, negative, general psychopathology and total scales of the PANSS were respectively 22 (s.d. 5), 19 (9), 40 (40) and 81 (20). The mean (s.d.) CGI score was 5 (s.d. 1) (5 is 'markedly ill').

There were no significant correlations between, on the one hand, scores on any of the PANSS and CGI scales and BMI and, on the other hand, plasma lipid

Table I Plasma lipid peroxide, serum cholesterol, vitamin E and vitamin A levels, and vitamin E : cholesterol ratios, in patients and control subjects

\begin{tabular}{lcr}
\hline & $\begin{array}{c}\text { Patients }(n=30) \\
\text { Mean }(\text { s.d.) }\end{array}$ & $\begin{array}{c}\text { Control subjects }(n=30) \\
\text { Mean (s.d.) }\end{array}$ \\
\hline Lipid peroxide $(\mu \mathrm{mol} / \mathrm{l})$ & $0.50(0.16)$ & $0.54(0.23)$ \\
Cholesterol $(\mathrm{mmol} / \mathrm{l})$ & $4.63(0.80)$ & $4.98(0.91)$ \\
Vitamin E $(\mu \mathrm{mol} / \mathrm{l}) *$ & $20.96(4.96)$ & $23.47(4.70)$ \\
Vitamin A $(\mu \mathrm{mol} / \mathrm{l})$ & $2.08(0.49)$ & $2.12(0.62)$ \\
Vitamin E : cholesterol ratio & $4.50(0.90)$ & $4.72(0.88)$ \\
\hline
\end{tabular}

*Mean differences 2.51 ; $95 \% \mathrm{Cl} 0.41-5.02$; paired $t$-test: $t=2.05$, d.f. $=29, P=0.04$. peroxide and serum vitamin $\mathrm{E}$ and vitamin A levels, and the ratio of vitamin $\mathrm{E}$ to cholesterol.

There was a significant negative correlation between patients' BMI and total score on the negative syndrome sub-scale of the PANSS (Pearson's $r=-0.42$, $P=0.03)$. Examination of the seven individual items making up the negative sub-scale showed that the only significant correlation with BMI was "passive/apathetic social withdrawal" ( $r=-0.42, P=0.03)$.

\section{DISCUSSION}

The patients recruited to our study were 'typical' first-episode schizophrenia sufferers. The mean age of onset of illness, as defined by age at first admission, was in the late 20s; there were more men than women; the age of onset was earlier in the men than in the women; and the patients were markedly ill, as measured by the PANSS and CGI scales.

\section{Smoking}

Seventy-three per cent of the patients were smokers, a much higher figure than that found in the general population in southwest Scotland (28\%) (Waldron et al, 1995). The percentage of smokers among our sample of male patients (mean age 28 years) is also much higher than that found among men aged 25-34 in a national survey (34\%) (Bennett et al, 1996). It has long been known that cigarette smoking is common in patients with schizophrenia (Hughes et al, 1986), and we have also reported in a retrospective study that most such patients start to smoke before their illness begins (Kelly \& McCreadie, 1999), a finding confirmed in the present study.

\section{Lipid peroxidation}

We found no difference between patients and control subjects in plasma lipid peroxide levels, and therefore our results do not support the view that increased lipid peroxidation is associated with the schizophrenic illness per se. This result is in contrast to that of the only other study which has examined peroxidation in first-episode patients (Mahadik et al, 1998). We believe our results are more likely to be valid for two reasons. First, our patients were more closely matched with control subjects than those in that earlier study; matching variables included gender, age, smoking and 
dietary status; also, the living arrangements were very similar in both groups. Second, mean plasma levels of lipid peroxide reported in that study, using the same method as ours (Young \& Trimble, 1991), were, for control subjects and patients respectively, $2.35 \mu \mathrm{mol} / \mathrm{l}$ (s.d. 0.78 ) and $5.16 \mu \mathrm{mol} / \mathrm{l}$ (s.d. 1.85), five and ten times as high as those in our study and compared with the mean levels reported in the description of the original HPLC method (Young \& Trimble, 1991). Reference values for most HPLC methods are $<1.5 \mu \mathrm{mol} / 1$ (Rumley $\&$ Paterson, 1998). It may be that there are methodological differences between the laboratory of Mahadik and colleagues and our laboratory.

Patients who smoked had higher lipid peroxide levels than those who did not. Smoking is a source of free radicals (Church $\&$ Pryor, 1985). As most of our patients were already smoking when first admitted to hospital, and as the prevalence of smoking among them was very much higher than in the general population, it could be argued that there is indeed increased lipid peroxidation in patients with schizophrenia, compared with the general population, but that it is mediated by smoking.

\section{Vitamin $E$ and diet}

Serum vitamin E levels were lower in patients than in control subjects and vitamin $\mathrm{E}$ : cholesterol ratios were low in both patients and control subjects; only $23 \%$ of patients had a ratio greater than 5 , a level which is said to be necessary to protect against heart disease (Hense et al, 1993). Diet in Scotland is generally poor and contributes to the high death rate from cardiovascular disease, the highest in the Western world (Scottish Office, 1993). The dietary choices of our first-episode patients probably reflect those of the general population. We have recently reported, however, that a group of chronic schizophrenic patients in south-west Scotland have an even worse diet than well-matched controls in terms of fibre, fruit and vegetable and antioxidant intake (McCreadie et al, 1998).

\section{Body mass index}

Patients had a lower BMI than controls, who were representative of the general population in south-west Scotland where $41 \%$ of males and $52 \%$ of females are either overweight or obese (Dumfries and Galloway Public Health Department, 1990). Why patients weigh less is not clear.

\section{CLINICAL IMPLICATIONS}

- The high prevalence of smoking and associated increased lipid peroxidation in first-episode patients with schizophrenia has long-term health implications.

- Low serum vitamin E levels suggest an unsatisfactory diet.

Dietary intervention with patients is necessary.

\section{LIMITATIONS}

- The number of patients and controls ( $n=30$ in each group) was small.

The subjects, both patients and controls, were Scottish; the Scottish diet is known to be poor.

Increased lipid peroxidation was not associated with the severity of mental state.

Correspondence: Dr Robin McCreadie, FRCPsych, Department of Clinical Research, Crichton Royal Hospital, Dumfries DGI 4TG. Tel. +44 1387 244000; Fax. +44 1387 257735; e-mail: rgmcreadie_crh@compuserve.com

(First received 19 July 1999, final revision 17 September 1999, accepted 21 September 1999)

However, there was a negative correlation between BMI and negative symptoms of schizophrenia; perhaps withdrawn, apathetic patients are less motivated to go out and buy the sorts of food their non-affected peers are purchasing. Chronically ill patients in south-west Scotland have been found to be overweight (McCreadie et al, 1998) probably as a result of antipsychotic medication (Stanton, 1995).

Schizophrenia sufferers die early (Mortensen \& Juel, 1990). We have shown in the present study that at the start of the illness there is both a high prevalence of smoking, associated with increased lipid peroxidation, and low serum vitamin $\mathrm{E}$ levels. Some of these adverse factors are likely to persist throughout the illness and may contribute to the early death of patients with schizophrenia. We now plan to make a more detailed assessment of the dietary habits of first-episode patients and embark upon dietary intervention studies.

\section{ACKNOWLEDGEMENTS}

The following are the members of the Scottish Schizophrenia Research Group: Crichton Royal Hospital, Dumfries: Dr R. G. McCreadie, Dr J. R. Paterson, Ms C. Blacklock, Mr D.Wiles, Dr D. J. Hall, Dr J. Graham, Dr S. McDonald, Dr G. Morrison, Dr A. Mitchell, Dr J. Allardyce; Leverndale Hospital, Glasgow: Dr J. McKane, Dr M. Hughson; Gartnavel Royal Hospital,
Glasgow: Dr C. Kelly, Dr M. Connolly; Woodilee Hospital, Glasgow: Dr M. Turner, Dr D. Patience, Dr J. Crean, Dr A. Yousef; Bellsdyke Hospital, Larbert: Dr K. Brown, Dr S. McCallum; Craig Dunain Hospital, Inverness: Dr A. Hay.

\section{REFERENCES}

American Psychiatric Association (1994) Diagnostic and Statistical Manual of Mental Disorders. (4th edn) (DSM-IV). Washington, DC: APA.

Bennett, N., Jarvis, L., Rowlands, O., et al (1996) Living in Britain. Results from the 1994 General Household Survey. London: Office of Population Censuses and Surveys.

Catignani, G. L. \& Bieri, J. G. (1983) Simultaneous determination of retinol and alpha-tocopherol in serum or plasma by liquid chromatography. Clinical Chemistry, 29, 708-712.

Chignell, C. F., Motten, A. G. \& Buettner, G. R. (1985) Photoinduced free radicals from chlorpromazine and related phenothiazines: relationship to phenothiazine-induced photosensitisation. Environmental Health Perspectives, 64, 103-110.

Church, D. F. \& Pryor, W. A. (1985) Free radical chemistry of cigarette smoke and its toxicological implications. Environmental Health Perspectives, 64 III-126.

Dumfries and Galloway Public Health Department (1990) Health and Lifestyle Survey. Dumfries: Dumfries and Galloway Health Board.

Guy, W. (1976) ECDEU Assessment Manual for Psychopharmacology. Revised DHEW Pub. (ADM). Rockville, MD: National Institute for Mental Health.

Hense, H.W., Stender, M., Bors, W., et al (1993) Lack of an association between serum vitamin $E$ and 
myocardial infarction in a population with high vitamin $\mathrm{E}$ levels. Atherosclerosis, 103, 21-28.

Hughes, J. R., Hatsukami, D. K., Mitchell, J. E., et al (1986) Prevalence of smoking among psychiatric outpatients. American Journal of Psychiatry, 143, 993-997.

Kay, S. R., Fiszbein, A. \& Opler, L. A. (1987) The positive and negative syndrome scale (PANSS) for schizophrenia. Schizophrenia Bulletin, 13, 26I-276.

Kelly, C. \& McCreadie, R. G. (1999) Smoking habits, current symptoms and premorbid characteristics of schizophrenic patients in Nithsdale, Scotland. American Journal of Psychiatry, 156, 175I-1757.

Lohr, J. B. (1991) Oxygen radicals and neuropsychiatric illness. Archives of General Psychiatry, 48, 1097-II06.
Mahadik, S. P. \& Mukhergee, S. (1996) Free radical pathology and antioxidant defense in schizophrenia: a review. Schizophrenia Research, 19, I-17.

$\ldots, \ldots$, Scheffer, R., et al (1998) Elevated plasma lipid peroxides at the onset of non-affective psychosis. Biological Psychiatry, 43, 674-679.

McCreadie, R. G., Macdonald, E., Blacklock, C., et a (1998) Dietary intake of schizophrenic patients in Nithsdale, Scotland: case-control study. British Medical Journal, 317, 784-785.

Mortensen, P. B. \& Juel, K. (1990) Mortality and cause of death in schizophrenic patients in Denmark. Acta Psychiatrica Scandinavica, 8I, 372-377.

Rumley, A. E. \& Paterson, J. R. (1998) Analytical aspects of antioxidants and free radical activity in clinical biochemistry. Annals of Clinical Biochemistry, 35 |8|-200.

Scottish Office (1993) The Scottish Diet: Report of a Working Party to the Chief Medical Officer for Scotland. Edinburgh: Scottish Office Home and Health Department.

Stanton, J. M. (1995) Weight gain associated with neuroleptic medications: a review. Schizophrenia Bulletin, 2I, 463-472.

Waldron, G., Chalmers, J., Bone, A., et al (1995) Health and Lifestyles in Dumfries and Galloway in 1995. Dumfries: Dumfries and Galloway Health Board.

Young, I. S. \& Trimble, E. R. (199I) Measurement of malondialdehyde in plasma by high performance liquid chromatography with fluorometric detection. Annals of Clinical Biochemistry, 28, 504-508. 